\title{
Zur Frage der Tuberkulindiagnostik.
}

\author{
Von \\ H. v. Hayek, \\ Innsbruck. \\ (Eingegangen am 2. Juli 1922.)
}

In dieser Frage ist sowohl von allzu kritiklosem Optimismus als auch von allzu bequemem und kurzsichtigem Negativismus viel gesündigt worden. Aber auch von Fachleuten, denen wir nach ihren sonstigen Leistungen unbedingt genügende praktische Erfahrung auf dem Tuberkulosegebiete und ernst zu nehmenden Arbeitswillen zusprechen müssen, erfährt diese Frage heute noch vielfach eine vollkommen entgegengesetzte Wertung, die auf der einen Seite in die kategorische Feststellung ,,von grundlegender Bedeutung“, auf der anderen Seite mit der absoluten Ablehnung, ,praktisch unbrauchbar" ausklingt. Solche gegensätzliche Werturteile lassen sich nur dadurch erklären, daß es sich um ein Arbeitsproblem handelt, bei dessen Lösung sich tatsächlich viel größere Schwierigkeiten entgegenstellen, als vielfach angenommen wird, und dem gegenüber es heute noch an einheitlichen Voraussetzungen fehlt.

Und damit ist bereits der Kernpunkt der ganzen Frage getroffen.

Die erste Voraussetzung für die praktische Verwertbarkeit der Tuberkulindiagnostik ist die logische Erkenntnis, daß auch den wechselvollen Verhältnissen der'Tuberkulinempfindlichkeit - wie jeder anderen spezifischen Reaktionsäußerung - Gesetzmäßigkeiten zugrunde liegen müssen, die mit dem Krankheitsgeschehen in einem Zusammenhang stehen.

Seit Jahren ist die diesbezügliche Literatur von ebenso sorglos als kraftvoll' hingeworfenen, entgegengesetzten Behauptungen erfüllt. „Die positive Tuberkulinreaktion" zeigt ,aktive" Tuberkulose an", dagegen ,,eine positive Tuberkulin reaktion sagt gar nichts, als daß der betreffende Organismus irgendeinmal mit Tuberkelbacillen in Berührung gekommen ist." "Die Tuberkulinreaktion ist absolut spezifisch" ", dagegen ,die Tuberkulinreaktion ist eine unspezifische Eiweißreaktion, die auch bei allen möglichen anderen Krankheitszuständen positiv ausfallen kann."

Was würden heute die Röntgendiagnostiker sagen, wenn auf ihrem Gebiet Ähnliches geleistet werden würde? ,Schatten im Lungengewebe zeigen ,alstive Tuberkulose an", dagegen ,diese Schatten.sagen gar nichts.". „Schatten im Lungengewebe zeigen spezifisch tuberkulöse Gewebsveränderungen an", dagegen "die gleichen Schatten sind bei allen möglichen anderen krankhaften und nicht krankhaften Zuständen zu finden." Logische Schlußfolgerung des Fernestehenden: die Röntgendiagnose ist für die Lungentuberkulose ohne praktische Bedeutung. 
Dieser Vergleich ist in vieler Hinsicht recht lehrreich. Auch bei der Einführung der Röntgendiagnostik gab es genug überschwängliche Erwartungen und vorschnelle Werturteile. Auch damals meinten viele, daß die Röntgenbilder mit einem Schlage alle diagnostischen Schwierigkeiten, die uns die Lungentuberkulose zu lösen gibt, beseitigen würden. Heute wissen wir, wie schwer. es war, wie viel theoretische und technische Arbeit geleistet werden mußte, bis es gelang, die Schatten des Röntgenbildes für eine differenzierte Diagnosenstellung verwertbar zu machen. Dieser Entwicklungsgang ist bei der Röntgendiagnose verhältnismäßig rasch erfolgt, denn hier steht als Voraussetzung unmittelbar das Substrat der pathologisch-anatomischen Gewebsveränderungen zur Verfügung.

Viel schwieriger und daher viel langsamer ist die Entwicklung biologischer Arbeitsprobleme, wo uns die fördernde unmittelbare Kontrolle des anatomischen Substrates fehlt, und wir erst auf Umwegen den Zusammenhang mit diesem suchen müssen.

Und hier finden wir für die praktische Verwertbarkeit der Tuberkulindiagnostik die zweite Voraussetzung gegeben: Jeder. Krankheitsprozeß beruht auf Störung der normalen biologischen Reaktionsvorgänge, Störungen, die früher oder später auch zu pathologisch-anatomischen Zustandsänderungen der Gewebe führen. Es muß daher auch zwischen diesen krankhaft veränderten Reaktionsvorgängen und den pathologisch-anatomischen Gewebsveränderungen ein gesetzmäßiger Zusammenhang bestehen.

Diese Gesetzmäßigkeiten zu suchen, die einerseits den wechselvollen Verhältnissen der Tuberkulinempfindlichkeit, andererseits ihren Beziehungen zu den pathologisch-anatomischen Zustandsänderungen bei der Tuberkulose zugrunde liegen, ist unsere nächste Aufgabe, wenn wir an eine praktische Verwertung des Tuberkulins zu diagnostischen Zwecken denken wollen.

Der begreifliche Wunsch, bei einer so wichtigen Sache, wie es die rechtzeitige Diagnose tuberkulöser Erkrankungen ist, mit einer einfachen Methode des Rätsels Lösung zu finden, hat manche dazu verleitet, diese Gesetzmäßigkeiten unter willkürlicher Ausschaltung tatsächlich gegebener komplizierender Fragen so einfach zu konstruieren, wie sie nach dem vorliegenden Tatsachenmaterial niemals liegen können. Und die ersten Widersprüche, die sich den vorschnell gezogenen, allzu primitiven Schlußfolgerungen entgegenstellten, haben wieder andere dazu gebracht, überhaupt an das Vorhandensein solcher Gesetzmäßigkeiten zu zweifeln.

Nach Ansicht der letzteren würde die Tuberkulinreaktion eine noch nie dagewesene Stellung in der ganzen medizinischen Forschung einnehmen: Reaktionsvorgänge - die zu dem Krankheitskomplex, dem sie entspringen, keine gesetzmäßigen Beziehungen zeigen.

„Die Tuberkulinreaktion" - ein böses oberflächliches Wort, das sich gelassen (obwohl von vielen [Much u. a.] mit Nachdruck abgelehnt) in die Tuberkuloseliteratur eingebürgert hat. Die Unsumme sinnfälliger Reaktionserscheinungen, die der tuberkulös-sensibilisierte Körper in wechselvollsten Erscheinungsformen auf die Einverleibung spezifischer Bacillensubstanzen zeigt - oder nicht zeigt, die je nach Dosierung, je nach den Zeitintervallen zwischen ihrer Wiederholung, je nach der Art der Einverleibung weitgehende Verschiedenheiten 
aufweisen - diese veränderlichen Reaktionserscheinungen, die unter genau den gleichen Versuchsbedingungen an Kranken „,mit sehr ähnlichem klinischen Befund“ die überraschendsten Widersprüche zeigen können, - Reaktionsvorgänge, deren Wesen, Entstehung und Deutung heute noch Gegenstand tiefgreifender theoretischer Meinungsgegensätze sind, sie sind „die Tuberkulinreaktion". Und diese reaktiven Vorgänge, die seit drei Jahrzehnten die Tuberkuloseforschung in Atem halten, die praktisch und theoretisch rastlos bearbeitet, kritiklos verwertet, in ihrer praktischen Brauchbarkeit vollkommen verworfen, immer wieder auferstehen, weil sie tief in das innerste Wesen tuberkulöser Krankheitsprozesse hineinleuchten, sie sagen jedem so viel, als er überhaupt in den Reaktionsvorgängen des tuberkulösen Körpers zu lesen versteht. Sie sind für den Optimisten, der sich kritiklos über alles hinwegsetzt, was gerade nicht in sein „System“ paßt, ohne weiteres des Rätsels Lösung. Sie sagen dem, der nicht $\mathrm{zu}$ lesen versteht, gar nichts. Und sie bieten dem, der über klinische Organbefunde und pathologisch-anatomische Zustandsänderungen hinaus bestrebt ist, die Entwicklung tuberkulöser Erkrankungen zu verstehen, um die schwere Aufgabe rechtzeitiger Diagnosen- und richtiger Prognosenstellungen zu leisten, manche praktische Möglichkeit, sich im Rahmen des ganzen vorliegenden Krankheitsbildes besser zurechtzufinden. Aber nicht „die Tuberkulinreaktion“ im üblichen Sinne bietet diese Möglichkeit, sondern die durch fleißige Übung und Erfahrung gewonnene Fähigkeit, die nach der Einverleibung spezifischer Reizkörper so mannigfaltig auftretenden sinnfälligen Reaktionserscheinungen - oder deren Ausbleiben - unter Vermeidung zahlreicher subjektiver und objektiver Fehlerquellen zur Klärung des vorliegenden Krankheitsbildes zu verwerten.

Die grundlegendsten Einzelheiten, die heute bereits den Charakter von Tatsachen erlangt haben, können hier nur in schlagwortmäßiger Kürze skizziert werden.

1. Es ist zu unterscheiden zwischen Reaktionserscheinungen in den eigentlichen Krankheitsherden, den Allgemeinreaktionen und den lokalen Hautreaktionen. Diese drei Reaktionsgruppen verlaufen zum Teil in klar erkennbarer Wechselbeziehung zueinander, zum Teil scheinbar recht unabhängig voneinander. Thre Gesetzmäßigkeiten vgl. v. Hayek „Das Tuberkuloseproblem“, J. Springer, Berlin.

2. Es gibt eine Abnahmeder Reaktionsempfindlichkeitnichtnurim ungünstigen Sinne, sondern auch im günstigen Sinne (für die stark ausgesprochene Abnahme der Reaktionsempfindlichkeit im günstigen Sinne habe ich den Verständigungsbegriff der „positiven Anergie“, im ungünstigen Sinne der ",negativen Anergie“ vorgeschlagen. Mit dem Begriff Anergie will ich dabei nicht mehr sagen als die Tatsache, da $\beta$ eben keine sinnfälligen Reaktionserscheinungen auftreten, und die Bezeichnungen positiv und negativ sollen nur die Tatsache eines zur Zeit gegebenen Gewinnes resp. Verlustes im prognostischen Sinne bezeichnen. Es ist daher weder die „positive Anergie“ ein logischer Widerspruch in sich, noch die „,negative Anergie“" eine Wiederholung des gleichen Gedankens. Man kann umschreiben: negative Anergie ist erschöpfte Reaktionsfähigkeit als Zeichen eines niederbrechenden Widerstandes; positive Anergie ist erhöhte Fähigkeit zur reaktionslosen Reizüberwindung). 
3. Beide sind durchaus relative Begriffe, in jedem Einzelfall abhängig von der Dosengröße und der speziellen Wirkungsart des verwendeten Präparates.

4. Gegen die negative Anergie zu sinkt bei der tertiären Phthise in der Regel zuerst die Empfindlichkeit der lokalen Hautreaktionen, während (durchaus ungünstig verlaufende) Herd- und Allgemeinreaktionen bis in die extremsten Krankheitsstadien erhalten bleiben können. Gegen die positive Anergie zu ist das Verhalten in der Regel umgekehrt, hier bleibt die Empfindlichkeit der lokalen Hautreaktionen in der Regel am längsten erhalten.

5. Starke Tuberkulinempfindlichkeit heißt heftiger biologischer Abwehrkampf: Dieser ist durchaus nicht gleichbedeutend mit „klinisch krank“,. wenn auch in der Regel Symptome eines subjektiven, wenig charakteristischen, allgemeinen Krankheitsgefühles bestehen.

6. Zwei Krankheitsstadien sind heute in ihrem biologischen Verhalten scharf umschrieben:

Die generalisierte (juvenile) Tuberkulose (Ranke, Hollo): sie zeigt in ihrer typischen Form jahrelang, auch bei vollkommener klinischer Gesundheit mehr oder minder starke Tuberkulinempfindlichkeit. Hier steht der Organismus noch in einem ständigen Abwehrkampf gegen die Entstehung der tertiären, isolierten Phthise.

Die isolierte Phthise (Ranke): der ganze Entwicklungsgang der Tuberkulose ist hier bereits durchgekämpft. Hier tritt in typischer Weise bei Fortschreiten der Dauerheilung zunehmende positive Anergie gegen Tuberkulin ein, während jeder neuerliche Rückfall die Tuberkulinempfindlichkeit wieder ansteigen läßt.

7. Bei der Bewertung einer fieberhaften Reaktion ist nicht die Höhe des Fiebers maßgebend, wie dies heute noch vielfach bewertet wird. Die Höhe des Fiebers sagt in weiten Grenzen nicht mehr als die individuelle Temperatureinstellung auf den gegebenen Reiz. Maßgebend ist vielmehr der Fiebertypus, vor allem das schnelle oder langsame Abklingen einer Reaktion (Maßstab für die Fähigkeit mehr oder minder vollkommener und rascher Reizüberwindung).

Es bedarf keines Wortes, daß bei der Tuberkulindiagnostik zur richtigen Beobachtung ambulanter Patienten eine gut durchgeführte Arbeitsorganisation unerläßlich ist, und daß man bei größerem Betrieb über ein gut geschultes Personal verfügen muß. Selbst aber muß man so weit sein, grobe Unwahrheiten in den Angaben der Patienten (falsche Temperaturmessungen usw.) als mit dem vorliegenden Krankheitsbild unvereinbar zu erkennen, und die wichtigsten Fehlerquellen (unspezifische Temperatursteigerungen, interkurrente Zwischenfälle verschiedenster Art usw.) bei seinen Schlußfolgerungen auszuschalten.

Bezüglich der Frage der Spezifität der Tuberkulinreaktion können wir heute wohl den Standpunkt einnehmen, daß ein absolut tuberkulosefreier Organismus nie auf Tuberkulin reagiert, daß aber, wie zahlreiche Veröffentlichungen gezeigt haben, im tuberkulosesensibilisierten Organismus (Krankenhausmaterial der Städte!!) auch nichttuberkulöse Krankheitsherde (wohl im Sinne einer Protoplasmaaktivierung, Weichhardt) nach Tuberkulininjektionen Herderscheinungen aufweisen können. Und dies darf uns in keiner Weise wundernehmen, denn wir sehen auch auf die verschiedensten physikalischen Reize hin, bei denen ja die Frage einer spezifischen Abstimmung gar nicht in Betracht kommt, unter ent- 
sprechenden Bedingungen ganz ähnliche Herderscheinungen auftreten. Es ist also sicher nicht berechtigt, einen Krankheitsherd, z. B. eine Gelenksaffektion, auf Grund einer reaktiven Reizung nach einer Tuberkulininjektion als ,tuberkulös" zu diagnostizieren. Eine solche Diagnose läßt sich aber häufig durch eine fortlaufende spezifische Reizbehandlung, wenn eine solche nach der Art des Falles an sich indiziert erscheint, recht eindeutig herausarbeiten.

Was endlich die Auslegung der einzelnen Reaktionserscheinungen betrifft, so werden noch immer ganz allgemein aus einzelnen Reaktionen viel zu weitgehende Schlüsse gezogen. Ich erwähne nur als einfachstes Beispiel, daß vielfach noch immer aus einer negativen Pirquetschen Reaktion auf fehlende Infektion geschlossen wird. Das ist durchaus unberechtigt. Ganz abgesehen von desolaten Fällen, wo die Hautanergie wirklich als Teilerscheinung einer negativen Anergie, eines Zusammenbruches der reaktiven Abwehrkräfte anzusprechen ist, und abgesehen von der bekannten Tatsache, daß die Pirquetsche Reaktion bei verschiedenen Hautexanthemen negativ wird, wissen wir heute, daß der positive oder negative Ausfall der Hautreaktionen in hohem Maße von den physikalischen Verhältnissen der Haut (Durchblutung, Ernährungszustand), von nervösen Momenten sowie von der Technik und der Wirkungsart und Dosierung des verwendeten Präparates abhängig ist.

Zahlreiche solche Veröffentlichungen liegen vor. So konnte auch ich namentlich während des Krieges vielfach in der schlecht ernährten, trockenen Haut von Rekonvaleszenten nach chronischen Darmaffektionen bei manifester Tuberkulose trotz guter Prognose, die auch durch den weiteren klinischen Verlauf sichergestellt wurde, auffallend schwache cutane und intracutane Reaktionen feststellen. Und ganz die gleichen Erfahrungen machte ich in meinem Ambulatorium während der.bösen Hungerjahre an stark unterernährten, sicher tuberkulösen Kindern. Ich erinnere mich an Fälle, wo unter mehreren sicher infizierten Geschwistern die kräftigeren starke Pirquetsche Reaktion zeigten, während die schwächlichen negativ oder nahezu negativ reagierten, ohne daß eine schwere Erkrankung folgte. Darunter Fälle, bei denen die Pirquetsche Reaktion schon nach einem mehrwöchigen Erholungsaufenthalt in einer Ferienkolonie u. dgl. stark positiv wurde.

Diese Hautanergie auf unspezifischer Grundlage stellt entschieden eine ungünstige Teilerscheinung dar. Sie ist aber weder im Sinne einer wirklichen spezifischen negativen Anergie und noch viel weniger als Zeichen fehlender Infektionen anzusprechen.

Bei der Beurteilung irgendeiner spezifischen Hautreaktion als Ausdruck spezifischer cellulärer Reaktionsvorgänge müssen wir, um nur irgendwelche diagnostisch-prognostische Schlußfolgerungen aus ihr im Rahmen des ganzen vorliegenden Krankheitsbildes ziehen zu können, uns ferner immer folgende vier typischen Möglichkeiten vor Augen halten:

schwere tuberkulöse Herderkrankung - schlechte Hautreaktivität; schwere tuberkulöse Herderkrankung - gute Hautreaktivität;

leichte tuberkulöse Herderkrankung - schlechte Hautreaktivität; leichte tuberkulöse Herderkrankung - gute Hautreaktivität. 
Sicher gibt uns aber die Pirquetsche Reaktion für einen allgemeinen Uberblick über das jugendliche Krankenmaterial und auch im Einzelfall bei entsprechender kritischer Wertung so manche wertvolle Aufschlüsse, so daß sich die geringe Mühe lohnt. Wer allerdings eine gesetzliche Behandlungspflicht auf die positive Pirquetsche Reaktion aufbauen will, der begeht einen ganz außerordentlich bedenklichen und unkritischen Fehler. Alle diese Reaktionen gehören in die Fand des Forschers und des praktischen Arztes, der sie kritisch zu werten versteht, nicht aber in die Hand von Bürokraten, wo sie mehr Unheil und Verwirrung als Nutzen stiften würden. Wenn ich für eine möglichst allgemeine Behandlung der Pirquet-positiven Kinder nach dem Petruschkyschen System warm eingetreten bin, so geschieht dies rein aus der Erwägung heraus, daß diese fortlaufende, billige und mühelose Reaktionsbehandlung, mit der in diesen Krankheitsstadien bei einigem Verständnis für die Sache kaum ein Schaden angerichtet werden kann, dem ganzen Wesen des gegebenen Krankheitsprozesses theoretisch ausgezeichnet angepaßt ist, und praktisch - ganz abgesehen von allen objektiv sicher schwer zu beurteilenden individuellen Erfolgen - große Vorteile in erzieherischer Hinsicht bietet. Und wenn ich sage auf ,Staatskosten", so meine ich damit noch lange nicht unter gesetzlich-bürokratischem Druck, sondern kostenlos an öffentlichen Stellen als wichtiges Propagandamittel für eine Tuberkulosebekämpfung auf wirklich breiter Grundlage.

Noch viel komplizierter liegen die Verhältnisse bei der subcutanen Tuberkulindiagnostik, wo wir alle drei Reaktionstypen: die Herd-, Allgemein- und Lokalreaktion in Betracht zu ziehen und ihre mannigfachen Wechselbeziehungen zu berücksichtigen haben.

Bei Herd- und Allgemeinreaktionen ist zunächst die Frage zu entscheiden, ob der Reaktionsablauf wirklich im spezifisch tuberkulösen Komplex zu suchen ist, oder ob es sich um einen sekundären Reaktionsablauf in einem unspezifischen Komplex handelt. Nur ganz klare und für den Kenner typische oder wiederholte Reaktionsqualitäten werden dabei ohne weiteres eindeutig.

Gibt es denn in der ganzen Medizin irgendeine diagnostische Reaktion, die - aus dem Zusammenhang herausgerissen - mit eindeutiger Sicherheit einen scharf zu differenzierenden Krankheitsprozeß ohne weiteres anzeigt?

Die Stärke einer Reaktion läßt durchaus nicht etwa einen Schluß auf besondere "Aktivität" des tuberkulösen Prozesses im Sinne einer schlechten Prognose zu. Wir finden vielmehr im Gegenteil sehr häufig bei gutartigen Prozessen (besonders generalisierte, juvenile Formen; nodöse Lungenherde mit Tendenz zur bindegewebigen Ausheilung) sehr kräftige Reaktionen, bei schweren, prognostisch ungünstigen Prozessen oft viel schwächere Reaktionen. Auch hier ist vor allem der Reaktionsablauf von Interesse. Rasches Abklingen starker Reaktionen ist ein Zeichen kräftiger Abwehrleistung, langsames Abklingen schwacher Reaktionen umgekehrt.

Es bedarf wohl weiter keines Wortes, daß die beriichtigte ,Tuberkulindiagnostik" mit Injektion von 1 oder $0,5 \mathrm{mg}$ Alt-Tuberkulin tatsächlich unbrauchbar - und nicht nur dies, sondern als sehr bedenklich durchaus abzulehnen ist. $1 \mathrm{mg}$ Alt-Tuberkulin kann für viele Tuberkulöse eine hundert- und tausendfach zu große Dosis sein, die zu sehr unerwünschten Reaktionserscheinungen führen 
kann, und sie kann in vielen anderen Fällen viel zu schwach sein, um sinnfällige Reaktionen zu geben.

Eine Tuberkulindiagnose, die über die Feststellung hinaus, daß es sich um einen tuberkulos-sensibilisierten Körper handelt, nach irgendwie praktisch verwertbaren diagnostischen und prognostischen Differenzierungen strebt, ist nur in Form einer richtig indizierten und richtig durchgeführten Tuberkulinbehandlung möglich. Das Wesen der Tuberkulinbehandlung ist ja eine Reizbehandlung, bei welcher der tuberkulöse Organismus zu gesteigerter Abwehrleistung gegen immer stärker erhöhte spezifische Reize trainiert werden soll - und auch mit bestem Erfolg trainiert werden kann. Das gelingt nur bei richtiger Technik, und erst die Frage, $o b$ dies gelingt, und unter welchen Reaktionsqualitäten dies gelingt, gibt uns die Möglichkeit, das Tuberkulin praktisch zu differenziert-diagnostischen und prognostischen Zwecken zu verwenden.

Nie dürfen wir bei so chronischen Krankheitsprozessen an momentanen Reaktionszuständen, die voll schwer zu überblickender Fehlerquellen sind, haften bleibén, sondern wir müssen lernen, langdauernde Entwicklungsgänge im Auge zu behalten. Für jeden, dem die technische Organisation einer solchen langdauernden Beobachtung seiner Patienten aus äußeren oder inneren Gründen nicht gelingt - ist die Tuberkulindiagnose, die diesen Namen wirklich verdient, vollkommen undurchführbar. Die „Tuberkulin-Schnelldiagnose“ in der Sprechstunde ist dabei der größte Schaden für die wertvolle Sache.

Den kritischen Ausführungen Junkers (Tuberkul.-Fürs. Bl. 1922, Nr. 5), der die spezielle Frage stellt, ob die Tuberkulindiagnose für die Feststellung der Behandlungsbedürftigkeit einer beginnenden Lungentuberkulose durch die Fürsorgestellen einen entscheidenden Wert hat, kann ich demnach nur vollkommen beipflichten, wie ich überhaupt mit jedem kritischen Standpunkt, sofern er nicht in einen bequemen und gedankenlosen Negativismus ausartet, viel eher zu einem Einverständnis komme, als mit dem Standpunkt so mancher „begeisterter" Anhänger der Tuberkulindiagnose.

Wer die Behandlungsbedürftigkeit des Krankenmaterials der Fürsorgestellen nach einer "positiven Tuberkulinreaktion“ stellen würde, der würde die Heilstätten nur sehr stark mit nicht Heilstättenbedürftigen, vollkommen gutartigen, vor allem juvenilen Tuberkuloseformen füllen, bei denen die Tuberkulinempfindlichkeit nichts anderes ist als der Ausdruck eines oft jahrelangen, notwendigen Abwehrkampfes, der durchaus nicht mit klinischer Erkrankung gleichzusetzen ist, und der mit und ohne Heilstätte in der Mehrzahl der Fälle eben jahrelang bestehen bleibt.

Einer Tuberkulindiagnostik an den deutschen Fürsorgestellen wird aber nach dem eben Gesagten um so weniger das Wort zu reden sein, da sich ja die deutschen Fürsorgestellen leider fast nie mit einer langdauernden spezifischen Kontrolle und Behandlung ihrer Patienten befassen.

So ist die Tuberkulindiagnostik nicht so einfach, wie manche sie vergeblich hinstellen, und nicht so unbrauchbar, wie andere glauben. Sie ist nur schwierig und erfordert, wie alles in der Medizin, erhebliches Wissen und entsprechende Utbung und praktische Erfahrung auf diesem Gebiete. Das ist bei anderen diagnostischen Methoden, die nach feineren Differenzierungen streben, nioht anders. 
Tausende von Röntgenapparaten sind heute auf dem Tuberkulosegebiete diagnostisch tätig, und doch muß ich nach den Erfahrungen meiner konsultativen Praxis; in der ich Röntgenplatten und Befunde verschiedenster Herkunft zu sehen bekomme, sagen, daß heute noch nicht allzuviele dieses kostspielige Verfahren zu genügend differenzierten Diagnosenstellungen zu verwerten verstehen.

Ich kann mit Befriedigung feststellen, da $\beta$ bei mir die Ergebnisse der Tuberkulindiagnose im Rahmen der ganzen "biologischen Entwicklungsdiagnose" mit den Ergebnissen guter und richtig gedeuteter Röntgenplatten sehr gut übereinstimmen - und das ist das wichtigste. Die biologische Entwicklungsdiagnose arbeitet langsamer, die Röntgendiagnose kostspieliger. Beide müssen bei richtiger Durchführung die prinzipiell gleichen Ergebnisse bringen. Beide bedürfen einer verständnisvollen Ergänzung durch Anamnese und durch das ganze klinische Krankheitsbild, in dessen Rahmen allein praktisch verwertbare diagnostische und prognostische Schlußfolgerungen im Einzelfall möglich werden. 